\title{
Acute Hemichorea as an Unusual Presentation of Internal Carotid Artery Stenosis
}

\section{Dong Wook Kim \\ Youngchai Ko \\ Sang Hyun Jang \\ Soo Jin Yoon \\ Gun-Sei Oh \\ Soo Joo Lee \\ Dong Joo Yun}

Department of Neurology, Eulji University Hospital,

Eulji University School of Medicine, Daejeon, Korea
Received October 29, 2012

Revised January 2, 2013

Accepted February 20, 2013

\section{Corresponding author}

Dong Joo Yun, MD

Department of Neurology,

Eulji University Hospital,

Eulji University School of Medicine,

1306 Dunsan 2-dong, Seo-gu,

Daejeon 302-779, Korea

Tel +82-42-611-3431

Fax +82-42-611-3858

E-mail_djy@eulji.ac.kr

- The authors have no financial conflicts of interest.
Involuntary movement associated with deep watershed ischemic lesions has been rarely reported. A 67-year-old woman presented with acute hemichorea on the left side. Magnetic resonance imaging showed acute infarcts in the anterior border zone. On perfusion studies, impaired cerebral blood flow was observed on the subcortical region sparing the basal ganglia. Cerebral angiogram confirmed severe stenosis in the right internal carotid artery. Her hemichorea gradually improved along with normalization of perfusion after carotid artery stenting with angioplasty. We suggest that impaired cerebral blood flow in critical watershed territories may be an important contributing factor in hemichorea associated with carotid occlusive disease.

Journal of Movement Disorders 2013;6:17-20

Key Words: Hemichorea, Carotid stenosis, Stents, Perfusion weighted MRI.

Hemichorea refers to forcible, flowing, involuntary arrhythmic movements on one side of the body. It may be associated with various conditions, such as cerebrovascular diseases, drugs, metabolic abnormalities, neurodegenerative diseases, and immunologic disorders. ${ }^{1}$ Chorea has been reported as the most common post-stroke movement disorder, usually associated with a lesion in the contralateral basal ganglia or related structures. ${ }^{2}$ Involuntary movement associated with deep watershed ischemic lesions that spare the basal ganglia has been rarely reported. We describe a patient with acute hemichorea associated with hemodynamic ischemia due to severe carotid artery stenosis, with reports of perfusion studies and discussion on its pathogenic mechanism.

\section{Case}

A 67-year-old woman with hypertension presented with acute left hemichorea involving the tongue, face, arm, and leg for the previous 3 days. She suddenly noticed the intermittent left orofacial and lingual dyskinesia with dysarthria, which occurred only when the patient tried to speak. The next day involuntary movements worsened and spread to the left arm and leg. It was nearly continuous although subsided during sleep. She had no history of convulsive disease, orthostatic hypotension, diabetes mellitus, or parkinsonism. She denied prior use of neuroleptics or oral contraceptives. She had no family history of involuntary movements or other neurologic disorders. A carotid bruit was auscultated over the right carotid bifurcation area. Neurological examination revealed repetitive, involuntary, irregular, purposeless, and non-rhythmic movements of the left tongue, face, arm, and leg. The patient was alert and oriented. There was no facial weakness. Her muscle strength, sensorium, and deep tendon reflexes were normal. She exhibited neither clonus nor a Babinski sign. Her gait was disturbed because of clumsy choreiform movements in the left distal leg. Neuropsychologic tests did not reveal bucco-linguo-facial apraxia, sensory extinction, and unilateral spatial neglect. Graphesthesia, stereognosis, and two-point discrimination were normal.

Blood tests including glucose, glycosylated hemoglobin A1c, thyroid and parathyroid function test, antistreptolysin O, rheumatoid factor, lupus anticoagulant, antiphospholipid anti- 
body, antinuclear antibody, antineutrophil cytoplasmic antibody, anti-Ro/La antibody, and peripheral blood morphology were unremarkable. 24-hour Holter monitoring revealed normal sinus rhythm and an echocardiogram showed no source of embolus. An electroencephalogram displayed continuous slowing in the right hemisphere suggestive of underlying cerebral dysfunction, but no epileptiform discharge was present.

Diffusion weighted MR images revealed acute small infarctions in primarily the right border zone between the anterior and middle cerebral artery (Figure 1). On perfusion MR scan, impaired cerebral blood flow and delayed mean transit time and time to peak were observed on the subcortical area around the infarcted lesions and no definite evidence of hemodynamic insufficiency was found in the basal ganglia (Figure 2A). Cerebral angiogram confirmed $90 \%$ stenosis with ath- erosclerotic ulcer in the right internal carotid artery (Figure $3 \mathrm{~A}$ ). The left internal carotid artery was mildly stenotic, and the intracranial arteries seemed unremarkable.

The patient was placed on aspirin and clopidogrel for acute stroke medication. Haloperidol and clonazepam have been administered, but the involuntary movements continued. Endovascular stent angioplasty was performed on the 16th day after symptom onset to treat the right carotid artery stenosis based on the presence of significant right hemispheric hypoperfusion without a large infarction (Figure 3B). A $9 \times 30 \mathrm{~mm}$ self-expanding close-cell type Wallstent (Boston Scientific, Natick, MA, USA) was deployed across the lesion with the proximal end in the distal common carotid artery. After stent delivery, post-deployment inflations were performed using a $6 \times 20 \mathrm{~mm}$ Aviator angioplasty balloon (Cordis, Miami, FL,
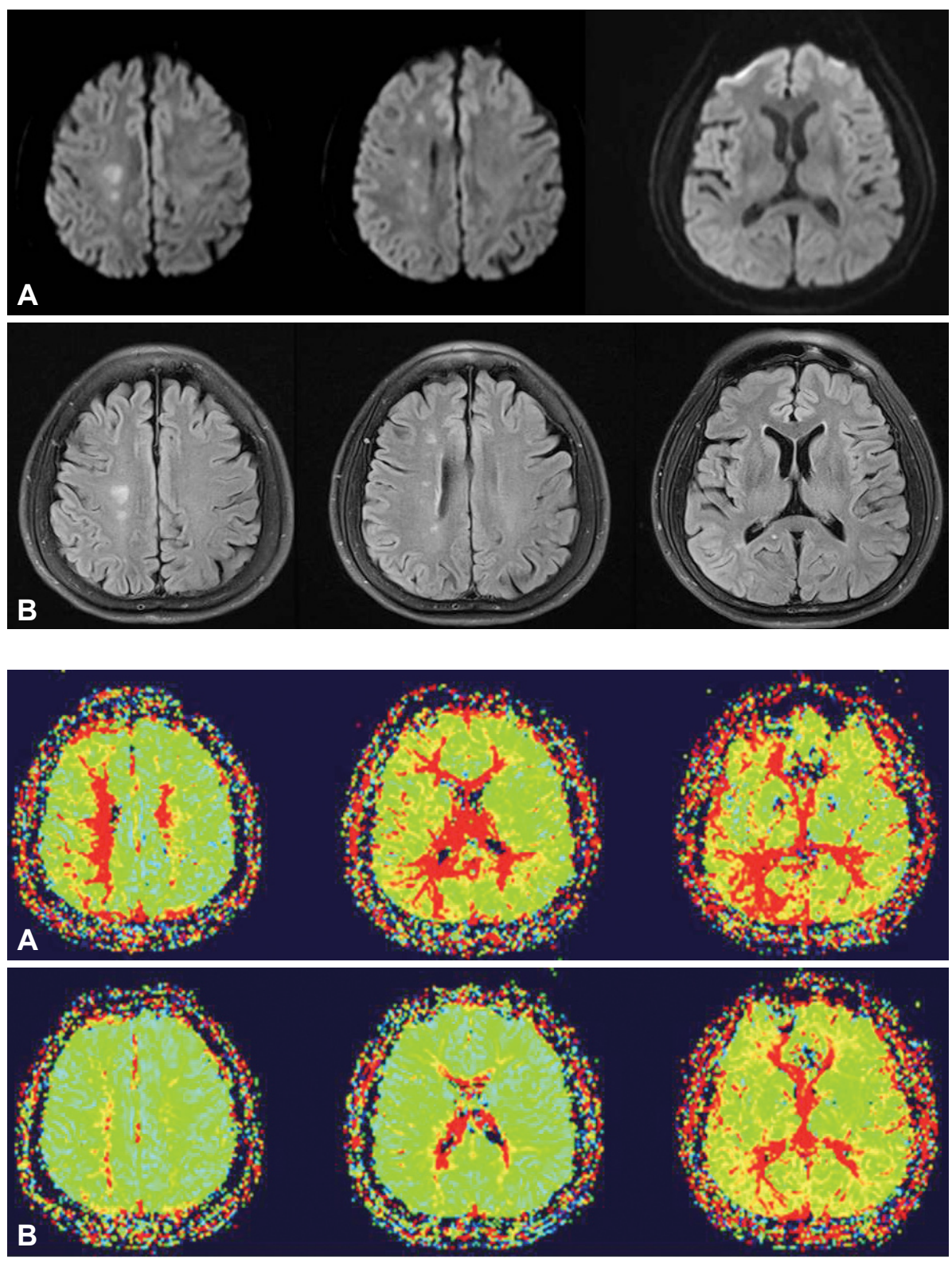

Figure 2. Pre-stenting perfusion-weighted MR scans $(A)$ show delayed mean transit on the subcortical area around the infarcted lesions. No definite evidence of hemodynamic insufficiency was found in the basal ganglia. Post-stenting follow-up perfusion images (B) demonstrate improvement of brain perfusion abnormalites in the right hemisphere. 
Figure 3. The lateral view of the right carotid artery angiogram $(A)$ demonstrates severe $(90 \%)$ stenosis with atherosclerotic ulcer in the right internal carotid artery. Post-stenting angiogram (B) shows successful recanalization of the carotid lesion.
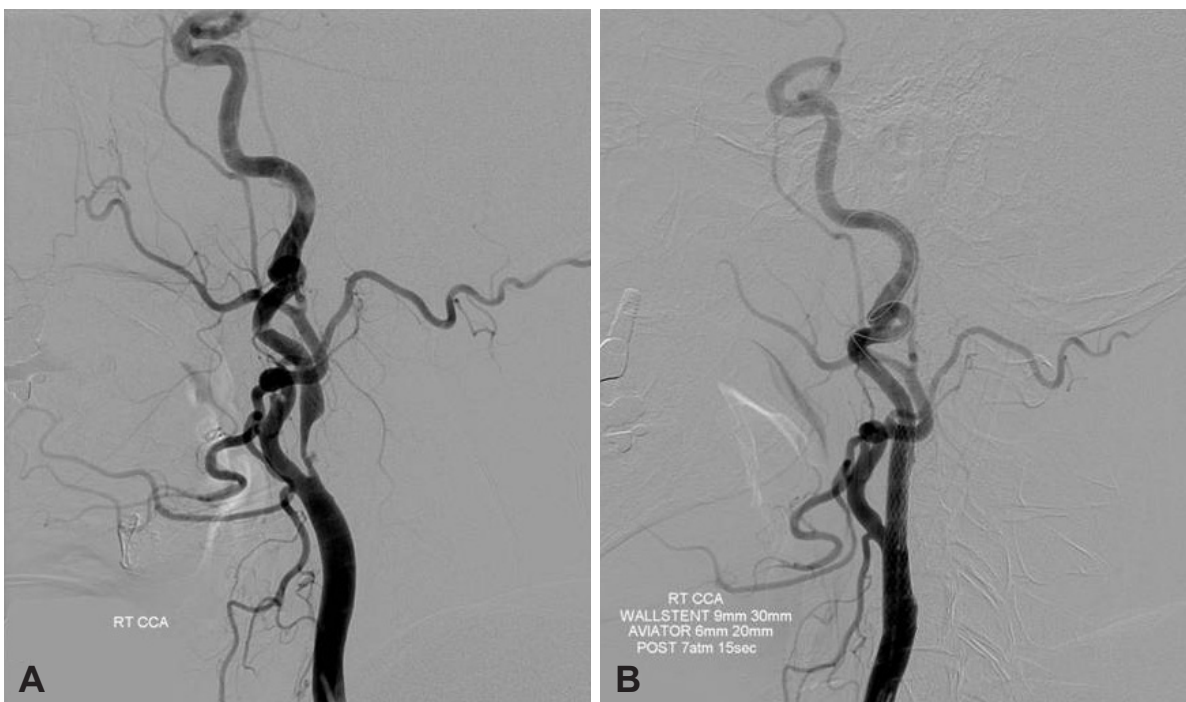

USA) due to significant underlying stenosis. Post-stenting perfusion MRI demonstrated improvement in the cerebral blood flow in the right hemisphere (Figure 2B). Her hemichorea gradually improved over the next few weeks along with normalization of perfusion after carotid artery stenting. At four month follow-up, she remained symptom free.

\section{Discussion}

We report an unusual case of hemichorea caused by internal carotid artery stenosis, which was reversed by reperfusion of the watershed territory through carotid angioplasty and stent placement.

Hyperkinetic movement disorders are uncommon in acute stroke, with a prevalence of $1 \%$ and an estimated incidence of $0.08 \%$ per year. ${ }^{3}$ Hemichorea is a very rare presentation of carotid occlusive disease to cause hemodynamic compromise in watershed territories. In searching the literature, we have found several reports of acute hemichorea or hemiballism associated with carotid artery occlusive disease..$^{4-9}$ However, these hyperkinetic movement disorders seem to be related to ischemic lesions in the basal ganglia or subthalamic nucleus in the majority of patients. There were few published cases examining the perfusion state of patients with hemichorea with no radiologic evidence of basal ganglia infarction. Subcortical white matter lesions also have been described, but the evidence of abnormalities of the basal ganglia could not be completely ruled out. ${ }^{5,8-10}$ In three reported series, ${ }^{5,8,9}$ cerebral angiography or perfusion studies demonstrated striatal hypoperfusion $^{5,8}$ and chorea subsided after carotid artery stenting ${ }^{8}$ or carotid endarterectomy, ${ }^{9}$ which supported functional changes in basal ganglia. However, the interruption of corticostriate fibers was rarely considered to be the cause of hemichorea due to lesions outside the basal ganglia. ${ }^{10}$

The anatomic basis of hemichorea seems to be nonspecific for any topographical location. Hemichorea can be found in lesions involving the caudate nucleus, putamen, thalamus, subthalamus, and subcortical white matter, which are located in the cortico-striato-pallido-thalamo-cortical feedback loop., ,11-14 $^{-1}$ In the present case, diffusion MRI showed infarcts in the anterior watershed region in which carotid stenosis may lead to the most severe reduction of cerebral blood flow. On perfusion MR studies, hemodynamic insufficiency was observed on the subcortical region sparing the basal ganglia, which supports the finding that the subcortical lesion interferes with neural connections between the basal ganglia and the cerebral cortex in the motor pathway without direct involvement of the basal ganglia. MR perfusion study has been a measure to identify potentially salvageable ischemic penumbra at risk progressing to infarction. It can also provide additional morphological information. Furthermore, MRI is more readily available and less time consuming compared to SPECT.

Prognosis seems to be favorable in hemichorea associated with subcortical white matter lesions, which may be caused by the functional disconnection rather than actual destruction of the basal ganglia-thalamo-cortical motor circuit. ${ }^{15}$ The involuntary movements in our patient disappeared along with an improvement in perfusion in subcortical region after carotid artery stenting. Impaired cerebral blood flow in critical watershed territories may be an important contributing factor in hemichorea associated with carotid stenosis. Recognition of this unusual form of carotid stenosis is crucial, since early diagnosis and appropriate treatment will reverse this deficit and reduce significantly the likelihood of a disabling stroke. Conservative treatments such as administration of antiepileptic agents or haloperidol may not be effective. ${ }^{16}$ Revascularization procedures such as carotid artery stenting may be helpful, which eliminate the source of emboli and the stenosis causing the insufficient circulations in the watershed areas.

In summary, our patient had hemichorea caused by the is- 
chemic state in the subcortical border zone due to severe carotid occlusive disease. This is derived from 1) the occurrence of severe carotid stenosis, affecting primarily the border zone between the anterior and middle cerebral artery distributions, 2) impaired cerebral blood flow on the subcortical area around the infarcted lesions on perfusion studies, and 3) complete resolution of hyperkinetic movements following correction of hemodynamic insufficiency by carotid stenting with angioplasty. This report may be helpful for the management of involuntary movements in patients with regional cerebral hemodynamic insufficiency. Further studies using perfusion images are necessary to clarify the pathogenic mechanism and anatomic basis of hemichorea associated with ischemic cerebrovascular disease.

\section{REFERENCES}

1. Cardoso F, Seppi K, Mair KJ, Wenning GK, Poewe W. Seminar on choreas. Lancet Neurol 2006;5:589-602.

2. Alarcón F, Zijlmans JC, Dueñas G, Cevallos N. Post-stroke movement disorders: report of 56 patients. J Neurol Neurosurg Psychiatry 2004;75: 1568-1574.

3. Ghika-Schmid F, Ghika J, Regli F, Bogousslavsky J. Hyperkinetic movement disorders during and after acute stroke: the Lausanne Stroke Registry. J Neurol Sci 1997;146:109-116.

4. Prick JJ, Korten JJ. Unilateral involuntary movements of acute onset in the adult, nine case reports and an alternative pathophysiological hypothesis. Clin Neurol Neurosurg 1988;90:321-327.

5. Fukui T, Hasegawa Y, Seriyama S, Takeuchi T, Sugita K, Tsukagoshi $\mathrm{H}$. Hemiballism-hemichorea induced by subcortical ischemia. Can J
Neurol Sci 1993;20:324-328.

6. Shimizu T, Hiroki M, Yamaoka Y, Kato S, Suda M, Ide K, et al. Alternating paroxysmal hemiballism-hemichorea in bilateral internal carotid artery stenosis. Intern Med 2001;40:808-812.

7. Morigaki R, Uno M, Suzue A, Nagahiro S. Hemichorea due to hemodynamic ischemia associated with extracranial carotid artery stenosis. Report of two cases. J Neurosurg 2006;105:142-147.

8. Pareés I, Pujadas F, Hernández-Vara J, Lorenzo-Bosquet C, Cuberas G, Munuera J, et al. Reversible hemichorea associated with extracranial carotid artery stenosis. J Neurol Sci 2011;300:185-186.

9. Galea I, Norwood F, Phillips MJ, Shearman C, McMonagle P, Gibb WR. Pearls \& Oy-sters: resolution of hemichorea following endarterectomy for severe carotid stenosis. Neurology 2008;71:e80-e82.

10. Barinagarrementeria F, Vega F, DelBrutto $\mathrm{OH}$. Acute hemichorea due to infarction in the corona radiata. J Neurol 1989;236:371-372.

11. Pantano P, Di Cesare S, Ricci M, Gualdi GF, Sabatini U, Di Piero V. Hemichorea after a striatal ischemic lesion: evidence of thalamic disinhibition using single-photon emission computed tomography: a case report. Mov Disord 1996;11:444-447.

12. Lownie SP, Gilbert JJ. Hemichorea and hemiballismus: recent concepts. Clin Neuropathol 1990;9:46-50.

13. Im SH, Oh CW, Kwon OK, Cho BK, Chung YS, Han DH. Involuntary movement induced by cerebral ischemia: pathogenesis and surgical outcome. J Neurosurg 2004;100:877-882.

14. Chang JH, Seo WK, Park MH, Lee JM, Kwon DY, Koh SB. Generalized chorea induced by an unilateral anterior cerebral artery territorial infarction. J Mov Disord 2009;2:37-39.

15. Chung SJ, Im JH, Lee MC, Kim JS. Hemichorea after stroke: clinicalradiological correlation. J Neurol 2004;251:725-729.

16. Kim JA, Jung S, Kim MJ, Kwon SB, Hwang SH, Kwon KH. A case of vascular hemichorea responding to topiramate. J Mov Disord 2009;2: $80-81$. 